\title{
Nonthermal ultrafast optical control of the magnetization in garnet films
}

\author{
Fredrik Hansteen,,${ }^{1,2} *$ Alexey Kimel, ${ }^{1}$ Andrei Kirilyuk, ${ }^{1}$ and Theo Rasing ${ }^{1}$ \\ ${ }^{1} I M M$, Radboud University Nijmegen, Toernooiveld 1, 6525 ED Nijmegen, The Netherlands \\ ${ }^{2}$ The Norwegian University of Science and Technology, 7491 Trondheim, Norway \\ (Received 8 July 2005; revised manuscript received 25 October 2005; published 24 January 2006)
}

\begin{abstract}
We demonstrate coherent optical control of the magnetization in ferrimagnetic garnet films on the femtosecond time scale through a combination of two different ultrafast and nonthermal photomagnetic effects and by employing multiple pump pulses. Linearly polarized laser pulses are shown to create a long-lived modification of the magnetocrystalline anisotropy via optically induced electron transfer between nonequivalent ion sites while circularly polarized pulses additionally act as strong transient magnetic field pulses originating from the nonabsorptive inverse Faraday effect. Due to the slow phonon-magnon interaction in these dielectrics, thermal effects of the laser excitation are clearly distinguished from the ultrafast nonthermal effects and can be seen only on the time scale of nanoseconds for sample temperatures near the Curie point. The reported effects open exciting possibilities for ultrafast manipulation of spins by light, and provide insight into the physics of magnetism on ultrafast time scales.
\end{abstract}

DOI: 10.1103/PhysRevB.73.014421

PACS number(s): 75.30.Gw, 78.47.+p, 78.20.Ls, 75.40.Gb

\section{INTRODUCTION}

Ultrafast magnetization dynamics has recently become one of the hottest topics in condensed matter physics, ${ }^{1-9}$ stimulated on the one hand by the ever increasing demand for increased speed of writing and retrieving magnetically stored information, and on the other hand by the development of ultrafast (femtosecond) laser sources. ${ }^{10}$ The latter allow for excitation of magnetic systems at much shorter time scales than fundamental quantities such as spin precession or spin-lattice relaxation times. This type of photoexcitation puts a medium in a strongly nonequilibrium state, where a conventional description of magnetic phenomena in terms of thermodynamics may no longer be valid. Therefore, in addition to the potential applications, ultrafast magnetization dynamics is a subject of extreme fundamental interest in the physics of magnetism.

The first ultrafast time resolved studies of the impact of laser pulses on the magnetization were done on $\mathrm{Ni}$ and $\mathrm{Fe}$ using picosecond laser pulses, but these were not successful in observing any magnetic effects up to the melting point of the samples. ${ }^{11,12}$ Later, using time-resolved spin-polarized photoemission as a probe of the magnetization Vaterlaus et al. succeeded in estimating the spin-lattice relaxation time in Gd films to be $100 \pm 80$ ps. ${ }^{13}$ In 1996 Beaurepaire et al. reported an observation of subpicosecond demagnetization in $\mathrm{Ni}$ induced by $60 \mathrm{fs}$ laser pulses. ${ }^{14}$ This ultrafast magnetic response was explained by an effective electron-spin interaction mechanism among the strongly nonequilibrium photoexcited electrons, leading to a rapid increase of the spin temperature and destruction of the magnetization. The observation triggered the interest of several groups and similar experiments confirmed the ultrafast demagnetization in $\mathrm{Ni}, \mathrm{Co}$, and other metallic systems. ${ }^{15-18}$ It was concluded that the magnetization follows the electron temperature with a delay between the electron excitation and the magnetic breakdown of no more than $50 \mathrm{fs}$. An experimental artifact was revealed by Regensburger et al. ${ }^{19}$ and Koopmans et al. ${ }^{20}$ who pointed out that the mageto-optical response does not always directly relate to the magnetization during the first few hundred femtoseconds as a result of hot electron effects. The speed of the true demagnetization was consequently reduced to $0.5-1 \mathrm{ps}$ and ascribed to an effective spin-lattice interaction. The significant role of artifacts in time-resolved magneto-optical experiments was also demonstrated in $a b$ initio calculations. ${ }^{21}$ Recently, however, it was shown that laser-induced spin dynamics indeed does take place during the initial electron thermalization with a characteristic time of about $50 \mathrm{fs},{ }^{22-24}$ thus again raising the question of the underlying mechanism. The complete interpretation of this rapid demagnetization is still not clear, partly because it is difficult to distinguish between different processes in metallic systems due to their complex electronic structure and the continuum of transitions. ${ }^{21,25,26}$

In addition to laser-induced demagnetization the triggering of spin waves by laser pulses have been studied. ${ }^{27-32}$ The equilibrium orientation for the magnetization is believed to be changed through thermal modulation of the magnetic anisotropy, and thus causing the magnetization to precess. In fact, for all of the above-mentioned experiments on metallic systems, the observed magnetic excitation was the result of optical absorption followed by a rapid temperature increase. Far more exciting is the possibility of ultrafast nonthermal control of magnetization by light, where a change in the magnetization is not simply the result of a temperature increase. It provides much greater freedom for the manipulation of the magnetization, and unwanted heating and possible material damage in devices can be avoided. The nonthermal influence of light on magnetization in metals has been predicted by theory, ${ }^{33}$ but many aspects of this are still subject to debate. ${ }^{21} \mathrm{~A}$ few experimental attempts to observe a nonthermal influence of light on metallic magnetic systems have been reported. ${ }^{31,34}$ However, no impact on the magnetization could be seen in the time after the optical pulse. We believe that this is partly due to the dominating thermal effect in metals, and to the unfortunate coincidence of several processes in the same narrow time window which hampers the analysis. ${ }^{35,36}$ 
Ferromagnetic semiconducting alloys, such as (Ga, Mn)As, have recently attracted much attention. ${ }^{37,38}$ In this type of material the ferromagnetism is mediated by the free carriers, and highly effective nonthermal control of the magnetization by light was reported in static measurements. ${ }^{39}$ However, these large values of the photoinduced magnetization have not been reproduced or confirmed by dynamic measurements with subpicosecond time resolution, ${ }^{40,41}$ and similar experiments have only shown thermal effects of light on the magnetic system. ${ }^{42,43}$ A number of difficulties are associated with this class of materials, and the understanding of their electronic, optical, and magnetic properties is currently very limited and controversial.

When seeking to improve our understanding of ultrafast spin dynamics and searching for nonthermal photomagnetic effects, dielectrics possess some significant advantages over metals and semiconductors. The phonon-magnon interaction responsible for thermal effects is much slower in dielectrics than in metals and therefore does not obscure the interpretation of the processes on shorter time scales. ${ }^{44}$ Moreover, the electron-spin scattering mechanism proposed in metals cannot exist in dielectrics due to the localized nature of their electronic states. And finally, magnetic dielectrics, in contrast to magnetic semiconductors, are characterized by a welldefined electronic structure and their optical and magnetic properties are well understood.

For about 50 years magnetic garnets have been one of the most popular types of magnetic dielectric materials for both research and applications. ${ }^{45,46}$ Their physical properties are well known and can be tailored over a wide range through chemical substitution and by varying their growth conditions. For decades they have been considered ideal model systems for the experimental and theoretical investigation of magnetic phenomena. Their optical absorption in the infrared spectral region is very low and they exhibit large magnetooptical effects caused by strong spin-orbit coupling. The linewidth of ferrimagnetic resonance in garnets can be extremely narrow, implying a very low damping of magnetic excitations. ${ }^{45}$ Additionally, static control of the magnetic anisotropy by light has been known for some time in this class of materials. For these reasons they seem to be ideal materials for the study of ultrafast spin dynamics in general and the search for nonthermal mechanisms for the optical control of magnetization in particular. ${ }^{47}$ Indeed, we have recently demonstrated ultrafast nonthermal control of both magnetic anisotropy and magnetization in garnet films. A brief account of this study was reported in Ref. 48

In this paper we present the results from an extensive study of ultrafast optical control of the magnetization in ferrimagnetic garnet films. Laser pulses of center wavelength $\lambda=805 \mathrm{~nm}$ and pulse width $100 \mathrm{fs}$ were used to both excite and to probe the magnetic response of the films. We demonstrate the existence of two different nonthermal photomagnetic effects, allowing for ultrafast control of both the magnetocrystalline anisotropy and the magnetization. Thermal effects are clearly distinguished from the nonthermal effects and can be observed on the time scale of several nanoseconds in the vicinity of the Curie temperature.

The paper is organized as follows: Experimental details including sample characteristics and experimental setup are given in Sec. II. In Sec. III we present and discuss the experimental results obtained from studies of laser-induced magnetization dynamics in garnets. This section is divided into four subsections describing, respectively, nonthermal optical control of magnetic anisotropy, nonthermal optical control of magnetization, ultrafast photomagnetic switching, and slow thermal effects that can be seen on longer time scales near the Curie point.

\section{EXPERIMENTAL}

The samples studied in this work are 4-8- $\mu$ m-thick ferrimagnetic garnet films of the composition $\mathrm{Lu}_{3-x-y} \mathrm{Y}_{x} \mathrm{Bi}_{y} \mathrm{Fe}_{5-z} \mathrm{Ga}_{z} \mathrm{O}_{12}$ grown on (001) oriented gallium gadolinium garnet (GGG) substrates by liquid phase epitaxy. All the results presented in this paper are from samples with $x=0.65, y=0.66$, and $z=1.15$, but the effects that we observe are also present in a whole series of samples with similar composition. Small amounts of $\mathrm{Pb}$ impurities are known to exist in these types of films due to the flux from which they are grown. The films have in-plane magnetization, $4 \pi M_{s}=550 \mathrm{G}$, Curie temperature $T_{\mathrm{C}}=400 \mathrm{~K}$, and show almost no domain activity. While bulk garnet crystals have cubic symmetry and possess a center of inversion, epitaxially grown thin garnet films seem to lack this center of symmetry, as has been demonstrated by the existence of a linear magnetoelectric effect ${ }^{49}$ and by strong optical second-harmonic generation. $^{50,51}$

The garnet films were studied in transmission using an all-optical pump and probe technique. Regeneratively amplified $100 \mathrm{fs}$ pulses of wavelength $\lambda=805 \mathrm{~nm}$ emitted from a Ti:Sapphire laser system at a repetition rate of $1 \mathrm{kHz}$ were split into two parts using a beam splitter. The most intense part (pump) was incident on the sample at near normal incidence. The magnetization dynamics induced by these pump pulses was followed in time by measuring the Faraday rotation $\theta_{\mathrm{F}}$ of the time delayed and much weaker probe pulses $\left(I_{\text {pump }} / I_{\text {probe }}>1000\right)$ as function of the variable pump-probe separation $\Delta t$. The Faraday angle $\theta_{\mathrm{F}}$ is proportional to the projection of the magnetization vector $\mathbf{M}$ along the wave vector $\mathbf{k}$ of the probe light:

$$
\theta_{\mathrm{F}} \propto \mathbf{M} \cdot \mathbf{k} .
$$

In our geometry (see Fig. 1) the measured Faraday rotation is therefore essentially a probe of the out-of-plane $M_{z}$ component of the magnetization. For sensitive detection of the magneto-optical Faraday rotation a balanced photodiode detector was used in combination with a box-car integrator. ${ }^{52} \mathrm{~A}$ synchronized optical chopper operating at $500 \mathrm{~Hz}$ was placed in the pump beam path, thereby blocking every second pump pulse and creating alternating pump-on and pumpoff conditions in the sample. For every pump-probe delay $\Delta t$ the pump-induced Faraday rotation was averaged over several excitation events by use of a lock-in amplifier. Pumpinduced changes of the optical transmittivity of the sample was recorded simultaneously with the Faraday rotation by measuring the intensity of the probe pulses in addition to their polarization rotation.

The linear optical absorption of these garnet films in the spectral region around $\lambda=805 \mathrm{~nm}(1.54 \mathrm{eV})$ is small 


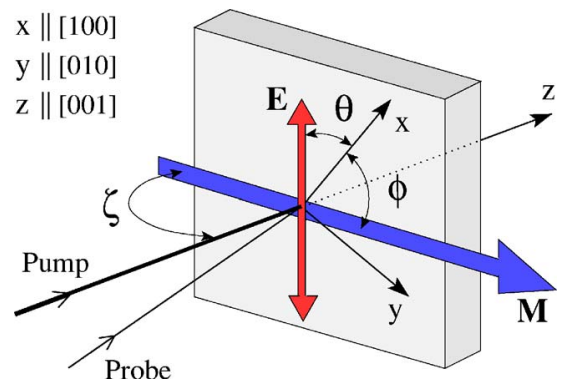

FIG. 1. (Color online) Experimental geometry. Pump and probe pulses were incident on the garnet film at near normal incidence. The magnetization $\mathbf{M}$ of the sample forms an angle $\zeta$ with the sample normal [001] and an angle $\phi$ with the crystallographic [100] $x$-axis of the film. For linearly polarized pump pulses the angle of the light's electric field $\mathbf{E}$ with respect to the sample $x$-axis is denoted $\theta$.

$\left(\alpha \approx 20 \mathrm{~cm}^{-1}\right)$ and mainly due to spin- and parity"forbidden" $d$ - $d$ transitions in the $\mathrm{Fe}^{3+}$ ions and a tail from higher energy charge transfer transitions at 2.8 and $3.4 \mathrm{eV} .{ }^{45,46}$ The magneto-optical properties of the material in the infrared part of the spectrum are dictated mainly by the tails of these high energy transitions. It is also well known that bismuth substitution strongly enhances the magnetooptical response. ${ }^{45,46}$

The Faraday rotation $\theta_{\mathrm{F}}$ measured with a saturating external field normal to the film plane is shown as function of the sample temperature in Fig. 2 for a $7.5-\mu \mathrm{m}$-thick garnet film. $\mathrm{M}(\mathrm{T})$ exhibits a second order phase transition with a critical exponent $\beta=0.414$ and a Curie temperature of $\mathrm{T}_{\mathrm{C}}=400 \mathrm{~K}$, both in agreement with previous studies of similar materials. ${ }^{45,46}$ From the hysteresis loop in Fig. 2(b) it can be seen that the sample exhibits no coercivity and has a large
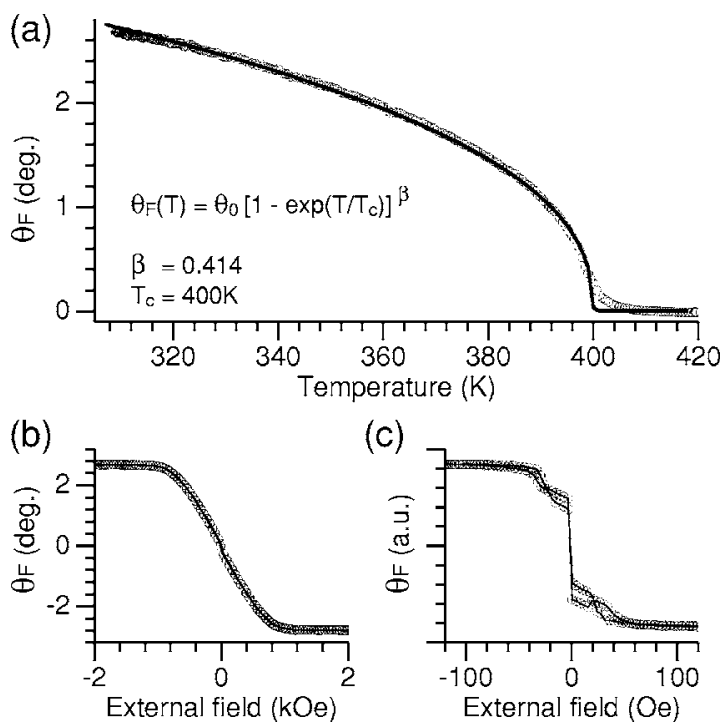

FIG. 2. Sample characteristics. (a) Measured Faraday rotation $\theta_{\mathrm{F}}$ at $\lambda=632.8 \mathrm{~nm}$ as a function of temperature with a saturating applied field $\mathbf{H}_{\text {ext }}$ normal to the film plane. (b) Hysteresis loop at $T=300 \mathrm{~K}$ measured with $\mathbf{H}_{\mathrm{ext}}$ normal to the film. (c) Hysteresis loop measured at a small angle of incidence with $\mathbf{H}_{\mathrm{ext}}$ in the sample plane, indicating the presence of anisotropy fields of about 50 Oe.
Faraday rotation of about $2.5^{\circ}$ at room temperature when saturated in the out-of-plane direction. The Faraday rotation measured at a small angle of incidence with the applied magnetic field parallel to the sample plane is shown by the hysteresis loop in Fig. 2(c). It gives an estimate of the film in-plane anisotropy $H_{\mathrm{a}}$ of about $50 \mathrm{Oe}$. Vibrating sample magnetometer (VSM) measurements reveal that this anisotropy has a fourfold symmetry in the plane.

Pump pulses of energy up to $20 \mu \mathrm{J}$ were focused to a spot diameter of about $200 \mu \mathrm{m}$ on the sample, corresponding to a photon density of approximately one photon per unit cell in the irradiated sample volume. The laser peak power density of about $10^{11} \mathrm{~W} / \mathrm{cm}^{2}$ is still well below the threshold for continuum generation in the garnet films. While the probe pulses always were linearly polarized, the polarization of the pump pulses could be varied using a Babinet-Soleil compensator. A magnetic field was applied either in the $x y$-plane of the sample, see Fig. 1, or at an angle with respect to the sample normal, thereby pulling the magnetization $\mathbf{M}$ out of the film plane $\left(\zeta<90^{\circ}\right)$. Additionally, the sample temperature could be controlled from room temperature up to well above the Curie point using a sample holder with a built-in heater and an electronic temperature regulator.

Unless otherwise explicitly stated, the results presented in the following section were all obtained at room temperature and with pump pulse energies near $20 \mu \mathrm{J}$.

\section{RESULTS AND DISCUSSION}

In this section, which is divided into four main parts, we present and interpret our experimental results from extensive studies of optically induced magnetization dynamics in garnet films. A remarkable amount of information about the underlying photomagnetic mechanisms can be obtained simply by analyzing time-traces of the precessional dynamics.

Coherent precession is the fastest known way to alter the direction of the macroscopic magnetization in a material. Phenomenologically the process is described by the LandauLifshitz equation of motion, ${ }^{53,54}$

$$
\frac{d \mathbf{M}}{d t}=-\gamma\left(\mathbf{M} \times \mathbf{H}_{\mathrm{eff}}\right) .
$$

It follows from this that the equilibrium orientation $(d \mathbf{M} / d t=0)$ for the magnetization $\mathbf{M}$ is along the direction of the effective magnetic field $\mathbf{H}_{\text {eff }}$ which is composed of the externally applied field $\mathbf{H}_{\text {ext }}$, the anisotropy field $\mathbf{H}_{\mathrm{a}}$, and the demagnetizing field $\mathbf{H}_{\mathrm{dem}}=-4 \pi M_{z} \hat{\mathbf{z}}$.

$$
\mathbf{H}_{\mathrm{eff}}=\mathbf{H}_{\mathrm{ext}}+\mathbf{H}_{\mathrm{a}}+\mathbf{H}_{\mathrm{dem}} .
$$

The key to optical manipulation of the magnetization lies in the the control of these fields by light.

Two different photomagnetic effects have been found to trigger coherent precession of the magnetization. In Sec. III A we present results showing that linearly polarized laser pulses create a long-lived modification of the magnetocrystalline anisotropy in garnet films. The effect is ultrafast and not of thermal origin, and it causes the magnetization to start precessing immediately after the photoexcitation. In Sec. 

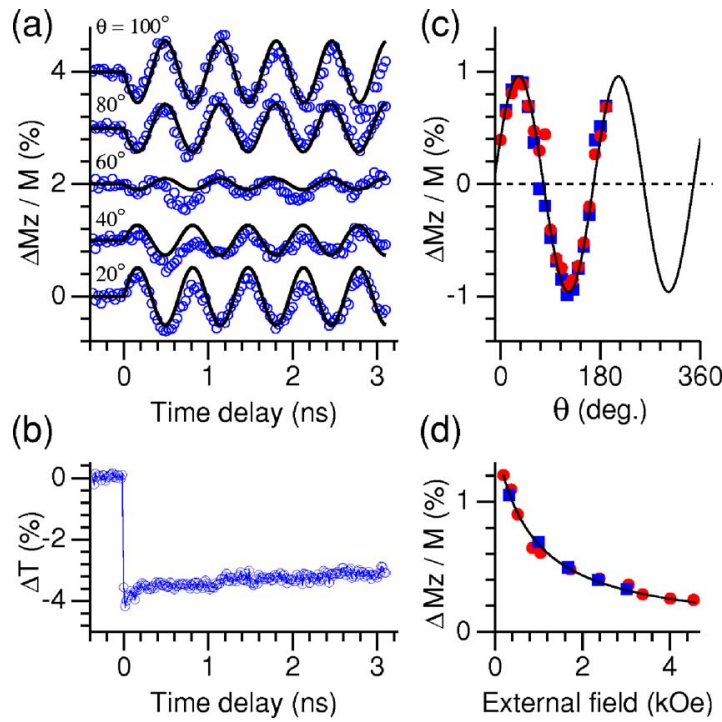

(d)

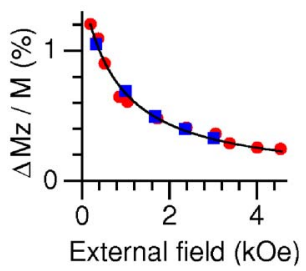

FIG. 3. (Color online) Coherent precession of the magnetization triggered by linearly polarized laser pulses. (a) Time dependence of the precession for different planes of pump polarization $\theta$, with an applied field of $\left|\mathbf{H}_{\text {ext }}\right|=350$ Oe in the plane of the sample. Circles represent measurements and solid lines simulations based on the Landau-Lifshitz equation. (b) Pump-induced change of the sample transmittivity $\Delta T$. (c) Precessional amplitude as a function of the plane of pump polarization. Round (red) and square (blue) symbols represent amplitudes extracted from measurements at $\pm \mathbf{H}_{\text {ext }}$. The solid line is a best fit. (d) Dependence of the precessional amplitude on the applied magnetic field $\mathbf{H}_{\text {ext }}$.

III B an additional effect of circularly polarized laser pulses on the magnetization is discussed along with the experimental results. It is found that these pulses act as strong axial magnetic field pulses during their presence in the sample. Also this effect is of nonthermal origin. These two photomagnetic effects can be combined as demonstrated in Sec. III $\mathrm{C}$ to achieve switching of the magnetization on a femtosecond time scale. Slow thermal effects of the laser pulses are also present and can be seen when the sample is held at temperatures close to the Curie point. These effects are discussed in Sec. III D.

\section{A. Photoinduced magnetic anisotropy}

\section{Experimental observations}

Applying an external magnetic field $\mathbf{H}_{\mathrm{ext}}$ in the plane of the sample (so that $\mathbf{M}$ is in plane, $\zeta=90^{\circ}$ ) and pumping with linearly polarized laser pulses, optically triggered precession of the magnetization $\mathbf{M}$ was observed, see Fig. 3(a). In the optical transmittivity of the sample Fig. 3(b), a sudden drop is seen which does not relax significantly within 3 ns. Intriguingly, the amplitude and phase of the precession in Fig. 3(a) was found to depend on the plane of polarization $\theta$ of the pump pulses as shown in Fig. 3(c). Negative values of the amplitude indicate precession of $\mathbf{M}$ with the opposite phase. Maxima of the precessional amplitude (of the opposite phase) were observed for every $90^{\circ}$ rotation of the polarization, and at some polarizations no precessional dynam-

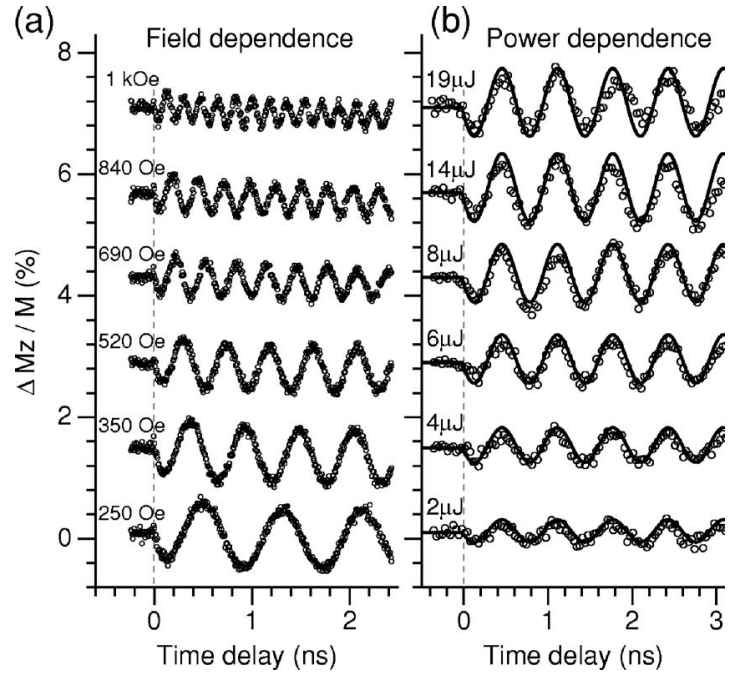

FIG. 4. Time traces of the precession excited by linearly polarized laser pulses (a) for different values of the in-plane applied magnetic field $\mathbf{H}_{\text {ext }}$ at a pump power of $20 \mu \mathrm{J} /$ pulse, and (b) for different pump powers with $H_{\mathrm{ext}}=300 \mathrm{Oe}$.

ics was triggered. From this dependence on pump polarization it is evident that the underlying effect must be nonthermal. An ultrafast heating effect would only reduce the magnitude of the magnetization and the anisotropy field independently of the pump polarization. Heating effects thus cannot be responsible for triggering magnetization dynamics that exhibit polarization dependence of the type that we observe in Fig. 3.

It is also interesting to note that $\mathbf{M}$ always starts its precessional motion by moving normal to the film plane along the $\pm \hat{z}$ direction. This follows from the initial phase of the measured signal in Fig. 3(a) which always starts from the inflection point where $M_{z}$ is changing most rapidly. From the Landau-Lifshitz equation [Eq. (2)] it can be inferred that immediately after the photo excitation both $\mathbf{M}$ and $\mathbf{H}_{\text {eff }}$ are in the film plane but not parallel to each other. Consequently, the observed magnetization dynamics must be due to an ultrafast change of the magnetization $\delta \mathbf{M}$, the anisotropy field $\delta \mathbf{H}^{\mathrm{a}}$, or a combination of the two, that effectively creates an in-plane angular displacement $\Lambda=\angle\left(\mathbf{M}, \mathbf{H}_{\text {eff }}\right)$ between $\mathbf{M}$ and $\mathbf{H}_{\text {eff. }}$. It is possible to distinguish between these possibilities by analyzing the precession amplitude $\Lambda$ as function of the applied field. The result is shown in Figs. 3(d) and 4(a). If triggered by an ultrafast rotation of the magnetization $\mathbf{M} \rightarrow \mathbf{M}+\delta \mathbf{M}$, the amplitude $\Lambda$ of the subsequent precession should be independent of the strength of the applied magnetic field as $\angle\left(\mathbf{M}, \mathbf{H}_{\text {eff }}\right)$ does not depend on $\mathbf{H}_{\mathrm{ext}}$. However, if precession is caused by a change in the effective field through a photoinduced anisotropy field $\delta \mathbf{H}^{\mathrm{a}}$, the precession amplitude $\Lambda$ is expected to decrease with increasing applied magnetic field as

$$
\Lambda=\angle\left(\mathbf{H}_{\mathrm{eff}}, \mathbf{H}_{\mathrm{eff}}+\delta \mathbf{H}^{\mathrm{a}}\right) \propto \frac{1}{\left|\mathbf{H}_{\mathrm{ext}}+\mathbf{H}_{\mathrm{a}}\right|}
$$

which is valid for small amplitude precessions. As shown by the fitted curve in Fig. 3(d) (solid line) the measurements 


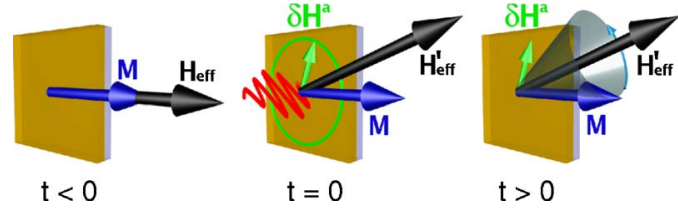

FIG. 5. (Color online) Graphical illustration of the process of photoinduced magnetic anisotropy caused by linearly polarized laser excitation and the subsequent precessional dynamics.

exhibit the exact behavior that one expects for a photoinduced anisotropy field $\delta \mathbf{H}^{\mathrm{a}}$. Based on the precession amplitude, the magnitude of the photoinduced field can be estimated to $\delta H^{\mathrm{a}}=0.5$ Oe for the present geometry $\left(\zeta=90^{\circ}\right)$. A graphical illustration of the excitation process and the subsequent precession is shown in Fig. 5.

For the present geometry, with the applied field in the plane of the film, changing the polarity of the magnetic field $\mathbf{H}_{\text {ext }}$ does not affect the measured signal for any given polarization of the pump. The fact that the precession phase and amplitude are both unaffected by reversing the polarity of the external field [see Fig. 3(c)] shows that $\delta \mathbf{H}^{\mathrm{a}}$ must be odd with respect to $\mathbf{M}$; when changing the polarity of the external field both $\mathbf{M}$ and the anisotropy field $\mathbf{H}_{\mathrm{a}}$ in Eq. (3) change sign. It then follows from Eq. (2) that the photoinduced $\delta \mathbf{H}^{\mathrm{a}}$ also must change sign, i.e., $\delta \mathbf{H}^{\mathrm{a}} \rightarrow-\delta \mathbf{H}^{\mathrm{a}}$ in order to give rise to the same signal.

By applying the external field at an angle the magnetization can be tilted out of the film plane $\left(\zeta<90^{\circ}\right)$. The actual angle $\zeta$ that the magnetization makes with the film normal is determined by the balance between the applied field, the anisotropy field, and the demagnetizing field. When pumping with linearly polarized laser pulses in this configuration, a larger amplitude precession was observed, see Fig. 6(a). This precession is superimposed on a slowly decaying exponential
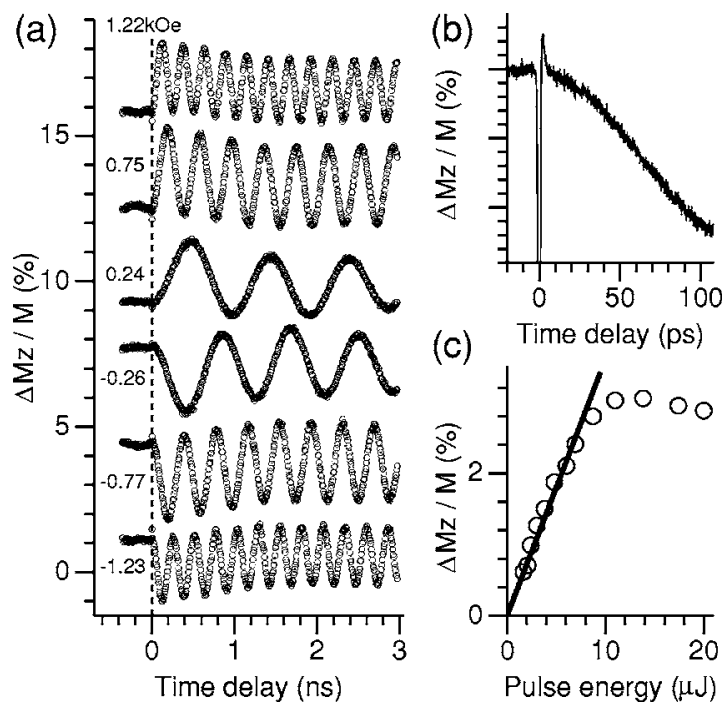

FIG. 6. (a) Precession of the magnetization following excitation with linearly polarized light for different values of the magnetic field applied at an angle of about $45^{\circ}$ with the sample normal. (b) The excitation shown on a finer time scale. (c) Dependence of precession amplitude on the excitation pulse energy. background caused by the relaxation of the photoinduced anisotropy. In contrast to the in-plane applied field geometry (where $\zeta=90$ ), the initial phase of the precession in Fig. 6(a) reveals that for $\mathbf{M}$ tilted out of the film plane $\left(\zeta<90^{\circ}\right)$ the initial motion of $\mathbf{M}$ is nearly parallel to the film plane. This implies that the laser-induced $\delta \mathbf{H}^{\mathrm{a}}$ is directed essentially along the $z$-direction. The dependence of the precession amplitude and phase on the polarization of the pump pulses becomes gradually smaller as $\mathbf{M}$ is tilted further out of the film plane. At about $\zeta=60^{\circ}$, all polarization dependence is practically gone and changing the polarity of the external field gives a near $180^{\circ}$ phase shift in the measured signal. The diminishing influence of the pump polarization is caused by the dominating $z$ component of $\delta \mathbf{H}^{\mathrm{a}}$, and will be discussed further in Sec. III A 3. From the precession amplitude in Fig. 6 the strength of the photoinduced anisotropy field is estimated to $\delta H^{\mathrm{a}}=1.5 \mathrm{Oe}$.

Laser heating effects in the sample, if present, are likely to be more pronounced in this geometry than in the in-plane field geometry as a thermal reduction of $\mathbf{M}$ also changes the equilibrium $\mathbf{H}_{\text {eff }}$ and leads to a reorientation of $\mathbf{M}$ along the $z$ direction. However, in our experiments the optical excitation of coherent spin waves is ultrafast [see Fig. 6(b)], much faster than the phonon-magnon interaction time which is about $1 \mathrm{~ns}$ in this material, ${ }^{45}$ and therefore cannot be of thermal origin. As will be discussed in Sec. III D, thermal effects can be seen on the time scale of a few nanoseconds when the sample is heated to temperatures near the Curie point.

Based on the results in Fig. 6(a) one can argue that the lifetime $\tau$ of $\delta \mathbf{H}^{\mathrm{a}}$ is longer than the time $t_{\exp }=3 \mathrm{~ns}$ accessible in this experiment. As the precession of $\mathbf{M}$ is always around the effective magnetic field $\mathbf{H}_{\text {eff }}^{\prime}=\mathbf{H}_{\text {eff }}+\delta \mathbf{H}^{\mathrm{a}}$, any relaxation of $\delta \mathbf{H}^{\mathrm{a}}$ should be visible in the time trace of the precession. Note in Fig. 6(a) how $\mathbf{M}$ precesses around an equilibrium $\mathbf{H}_{\text {eff }}^{\prime}$ that is different from the initial $t<0$ state. Some relaxation of $\mathbf{H}_{\text {eff }}^{\prime}$ can be seen (the slow overall change of the fast oscillating signal) but is not sufficient to restore the original equilibrium on the time scale of the experiment. This indicates that after $t_{\exp }=3 \mathrm{~ns} \delta \mathbf{H}^{\mathrm{a}}$ has still not decayed completely. Another observation that supports this conclusion is the photoinduced change in the sample transmittivity $\Delta T$ shown in Fig. 3(d), which also does not relax significantly during 3 ns.

There appears to be a linear relation between the precession amplitude and the pump power [Figs. 6(c) and 4(b)] up to pulse energies of almost $10 \mu \mathrm{J}$. At higher pulse energies the effect saturates completely. Based on the absorption coefficient the estimated density of absorbed photons is about one per hundred unit cells in the illuminated crystal volume. Saturation effects are therefore not expected unless they are caused by the presence of low concentration impurities. This will be discussed in more detail in the following section on the microscopic basis of the photomagnetic effect.

\section{Double-pump control of anisotropy}

In order to investigate the possibility of repeatedly modifying the anisotropy field on a time scale shorter than its relaxation time a double pump experiment was conducted. Using a Michelson interferometerlike configuration, the 


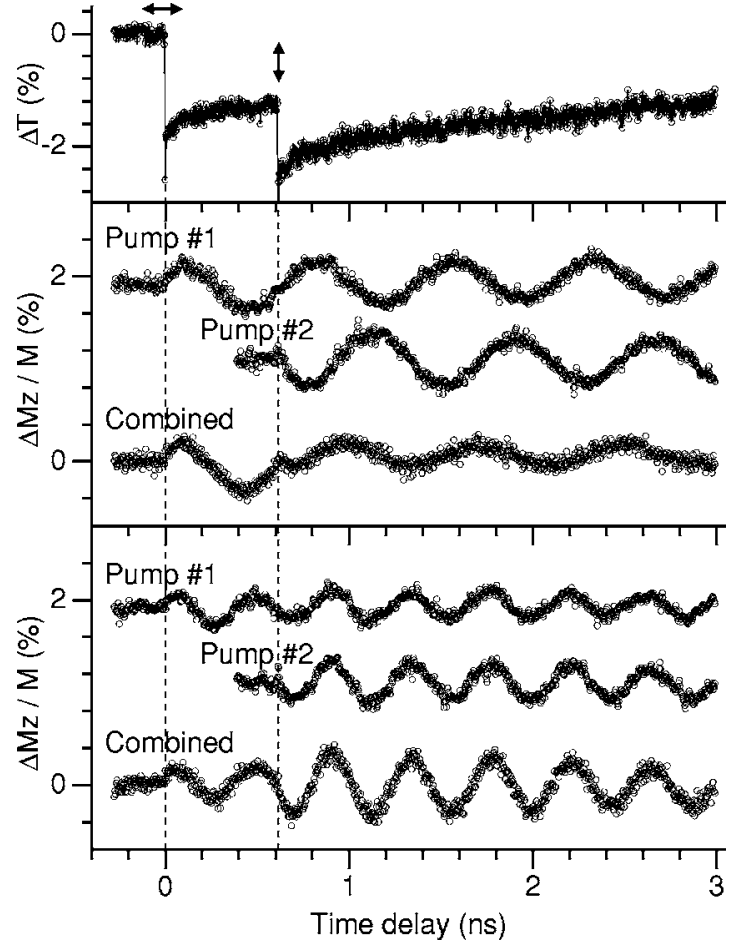

FIG. 7. A double pump experiment with two $6 \mu \mathrm{J}$ orthogonal linearly polarized pump pulses separated in time by approximately 600 ps. Timing with respect to the spin precession is done by varying the in-plane applied magnetic field and thereby the precession frequency. The top panel shows the photoinduced change of sample transmittivity. Partial quenching (middle panel) and amplification (lower panel) of the precession was achieved.

pump pulses were split into two with a beam splitter cube, and one part was delayed with respect to the other. A fixed time delay was used and the timing of the arrival of the second pump pulse with respect to the precessional dynamics was controlled by varying the precession frequency (applied field). By use of a quarter wave plate the linear polarization of the second pump pulse was set to be orthogonal with respect to the first one. A magnetic field was applied in the plane of the sample, and the dynamics triggered by the individual pump pulses was first recorded by blocking one of the pump pulses at a time. The results are shown in Fig. 7. The two orthogonally polarized pump pulses (denoted by pumps 1 and 2) trigger precession with the same amplitude and opposite phase, a result which was also known from Fig. 3 (c). When allowing both pump pulses to reach the sample the resulting dynamics (denoted combined) in the time after the second pump pulse ( $t>0.6 \mathrm{~ns})$ is almost identical to the sum of the response of the two individual pump pulses. If the second pump pulse arrives after approximately one full precessional period, as shown in the middle panel, it causes quenching of the subsequent dynamics. However, the timing was not accurate enough to completely quench the precession in the present case. If the second pump pulse arrives after one and a half periods it causes subsequent precession with twice the amplitude, as shown in the lower panel. The two pump pulses appear to act completely independently, indicating that we are operating in the regime of linear re- sponse (see Fig. 6). However, the experiment does not provide an answer to whether the long lived anisotropy $\delta \mathbf{H}^{\mathrm{a}}$ created by the first pump pulse is destroyed by the second pulse, or if the second pulse just creates additional anisotropy in the opposite direction.

In order to better understand our experimental results we describe in the following sections both a macroscopic phenomenological model and a microscopic one that accounts for the observed effects.

\section{Phenomenological model of photoinduced magnetic anisotropy}

In this section we give a macroscopic phenomenological description of the observed photoinduced magnetic anisotropy. The model is not concerned with the microscopic mechanism of the effect, but gives some insight into its symmetry properties.

The creation of a static magnetic field $\delta \mathbf{H}^{\mathrm{a}}(0)$ in the sample can be described as a combination of the nonlinear process of optical rectification ${ }^{55}$ and a linear magnetoelectric effect $^{56}$

$$
\delta H_{i}^{a}(0)=\chi_{i j k l} E_{j}(\omega) E_{k}(\omega) M_{l}(0) .
$$

Here $E$ is the electric field component of light and $M$ is the magnetization of the garnet film. The fourth rank polar tensor $\chi_{i j k l}$ has nonzero components for crystals of any symmetry. ${ }^{57}$

When taking the experimental geometry (Fig. 1) and the symmetry of $\chi_{i j k l}$ for the $4 \mathrm{~mm}$ point group of our samples into account, only four independent nonzero components of the tensor $\chi_{i j k l}$ remain;

$$
\begin{gathered}
A=\chi_{x x x x}=\chi_{y y y y}, \\
B=\chi_{x y x y}=\chi_{x x y y}=\chi_{y x y x}=\chi_{y y x x}, \\
C=\chi_{x y y x}=\chi_{y x x y}, \\
D=\chi_{z x x z}=\chi_{z y y z},
\end{gathered}
$$

and the vector components of the photoinduced anisotropy field are given by

$$
\begin{aligned}
& \delta H_{x}^{a} \propto E_{0}^{2} M_{s} \sin \zeta[(A+C) \cos \phi+(A-C) \cos 2 \theta \cos \phi \\
&+2 B \sin 2 \theta \sin \phi], \\
& \delta H_{y}^{a} \propto E_{0}^{2} M_{s} \sin \zeta[(A+C) \sin \phi-(A-C) \cos 2 \theta \sin \phi \\
&+2 B \sin 2 \theta \cos \phi], \\
& \delta H_{z}^{a} \propto E_{0}^{2} M_{s} D \cos \zeta .
\end{aligned}
$$

Here $\delta H_{i}^{a}$ is the photoinduced field along the $i$ direction, $i=\{x, y, z\}$ refers to the crystal axes of the sample, $\phi$ denotes the azimuthal angle between the sample $x$ axis and the projection of the magnetization vector on the film plane, and $\zeta$ is the angle between the film normal and the magnetization, as shown in Fig. 1.

From these equations one can see that if the magnetization $\mathbf{M}$ is in the film plane, the out-of-plane component $\delta H_{z}$ 
of the photoinduced anisotropy field does not contribute as $\cos \zeta=0$. This is in accordance with our experimental results from Fig. 3 which show an in plane $\delta \mathbf{H}^{\mathrm{a}}$. However, in order for the above equations to describe a field $\delta \mathbf{H}^{\mathrm{a}}$ consistent with the polarization dependence of the precession amplitude, shown in Fig. 3(c), the number of independent tensor components must be further reduced. The fact that there is no amplitude offset in the curve shown in Fig. 3(c) requires that $A=-C$ so that the first term in Eqs. (7) and (8) vanishes. Furthermore, the sinusoidal shape of the curve implies that $A=B$ and leaves us with only two independent components of the tensor $\chi_{i j k l}$,

$$
\begin{aligned}
A & =\chi_{x x x x}=\chi_{y y y y}=-\chi_{x y y x}=-\chi_{y x x y}=\chi_{x y x y} \\
& =\chi_{x x y y}=\chi_{y x y x}=\chi_{y y x x}, \\
D & =\chi_{z x x z}=\chi_{z y y z} .
\end{aligned}
$$

These additional equalities indicate that the $\chi_{i j k l}$ tensor has a higher symmetry than the garnet crystal. However, this does not violate Neumann's principle which states that the symmetry elements of any physical property of a crystal must include all the symmetry elements of the point group of the crystal. This does not prevent that property from having a higher symmetry than the crystal. The optically induced anisotropy field can now be written as

$$
\begin{gathered}
\delta H_{x}^{a} \propto A E_{0}^{2} M_{s} \sin \zeta[\sin 2 \theta \sin \phi+\cos 2 \theta \cos \phi], \\
\delta H_{y}^{a} \propto A E_{0}^{2} M_{s} \sin \zeta[\sin 2 \theta \cos \phi-\cos 2 \theta \sin \phi], \\
\delta H_{z}^{a} \propto D E_{0}^{2} M_{s} \cos \zeta .
\end{gathered}
$$

For the in-plane field geometry $(\cos \zeta=0)$ this describes a vector of constant length and with a direction depending on the angle $\phi$ of the magnetization with respect to the $x$-axis and the plane of polarization $\theta$ of the pump pulses. The $\delta H_{z}^{\text {a }}$ component accounts for the observed behavior in Fig. 6 with the applied field at an angle so that $\zeta<90^{\circ}$.

Computer simulations based on this simple model and the numerical integration of Eq. (2) exhibit good agreement with our experimental results both for the in-plane $\mathbf{H}_{\text {ext }}$ geometry shown in Fig. 3(a), and for the out-of-plane $\mathbf{H}_{\text {ext }}$ geometry in Fig. 6 (simulations are not shown). The latter indicate that the tensor component $D$ is larger than $A$ by a factor of 3 . This is not surprising in view of the symmetry distortion along the $z$ axis known to exist in films of this type. ${ }^{49,50,58}$

\section{Microscopic justification}

Photomagnetic effects are known to exist in garnets containing certain dopants, ${ }^{59,60}$ in particular Si and Co. ${ }^{61,62}$ Optically induced electron transfer between ions on nonequivalent sites in the crystal is believed to cause a change in the magnetocrystalline anisotropy due to a redistribution of ions. ${ }^{63}$ This effect is strong in crystals doped with elements that can assume different valence states, and where their contribution to the anisotropy is different. However, it has also been observed in undoped garnet samples containing $\mathrm{Pb}$ impurities ${ }^{64}$ which we believe is the case in our experiments.
The linear dependence of $\delta \mathbf{H}^{\mathrm{a}}$ on the pump power shown in Fig. 6 suggests that linear optical absorption is the dominating absorption process. The saturation of $\delta \mathbf{H}^{\mathrm{a}}$ at high pump intensities may be attributed to the $\mathrm{Pb}$ impurities. Divalent $\mathrm{Pb}^{2+}$ ions substitute trivalent $\mathrm{Lu}^{3+}$ ions on dodecahedral sites in the crystal and act as electron acceptors. This is a $p$-type doping which creates holes that are usually assumed to be located on iron ions in tetrahedral sites. ${ }^{46,65}$ To maintain overall charge neutrality in the crystal, some tetrahedrally coordinated trivalent iron ions change their valency to $4+$. Photoexcitation can induce a charge transfer between these $\mathrm{Fe}^{4+}$ ions and $\mathrm{Fe}^{3+}$ magnetic ions on octahedral sites, thus effectively "moving" the $\mathrm{Fe}^{4+}$ ions to sites with different symmetry, and thereby causing a change in the magnetic anisotropy. The low concentration of $\mathrm{Pb}$ impurities creates a limited number of photoactive ions and the photomagnetic effect can therefore be expected to saturate under intense illumination. An estimate for our sample shows that the illuminated volume of garnet film contains about $10^{12} \mathrm{~Pb}$ ions. An optical pulse of $20 \mu \mathrm{J}$ delivers $10^{14}$ photons from which about $1 \%$ is expected to be absorbed. This allows, in principle, for all of the photoactive ions to be excited and it is thus not surprising that saturation can occur at these pump intensities. The pump-induced change in transmittivity is also believed to be related to the photoexcitation of impurities. ${ }^{66}$

\section{B. Optical control of magnetization}

\section{Experimental observations}

Left- and right-handed circularly polarized laser pulses were used to excite the garnet film exposed to an in-plane applied magnetic field $\mathbf{H}_{\text {ext }}$. Precession of $\mathbf{M}$ with an opposite phase and different amplitude was triggered by pulses of helicity $\sigma^{+}$and $\sigma^{-}$, see Fig. 8 . Note also that the precession amplitude for $\sigma^{+}$is larger than the precession amplitude triggered by the linearly polarized pump pulses in the same geometry. As for linearly polarized pulses, the initial phase of the signal reveals that $\mathbf{M}$ initially moves along the $\pm z$ direction and therefore both $\mathbf{M}$ and $\mathbf{H}_{\text {eff }}$ are parallel to the film plane immediately after the photoexcitation.

In order to understand this result and the mechanism of excitation we analyze our model of the photoinduced anisotropy [Eq. (5)] for circularly polarized light $\mathbf{E}$ $=\left(E_{0} / \sqrt{2}\right)(\hat{x}+i \hat{y})$.

$$
\begin{gathered}
\delta H_{x}^{a} \propto A E_{0}^{2} M_{s} \sin \zeta \cos \phi, \\
\delta H_{y}^{a} \propto-A E_{0}^{2} M_{s} \sin \zeta \sin \phi, \\
\delta H_{z}^{a} \propto D E_{0}^{2} M_{s} \cos \zeta .
\end{gathered}
$$

We find that a photoinduced $\delta \mathbf{H}^{\mathrm{a}}$ may still exist which only depends on the direction $\phi$ of $\mathbf{M}$ with respect to the crystal axes. This is reasonable as $\theta$ has no meaning for circularly polarized light. For in-plane magnetization the photoinduced $\delta \mathbf{H}^{\mathrm{a}}$ is parallel to the film plane. However, it does not depend on the helicity of light and can therefore not account for the opposite phase of precession induced by the light of opposite 


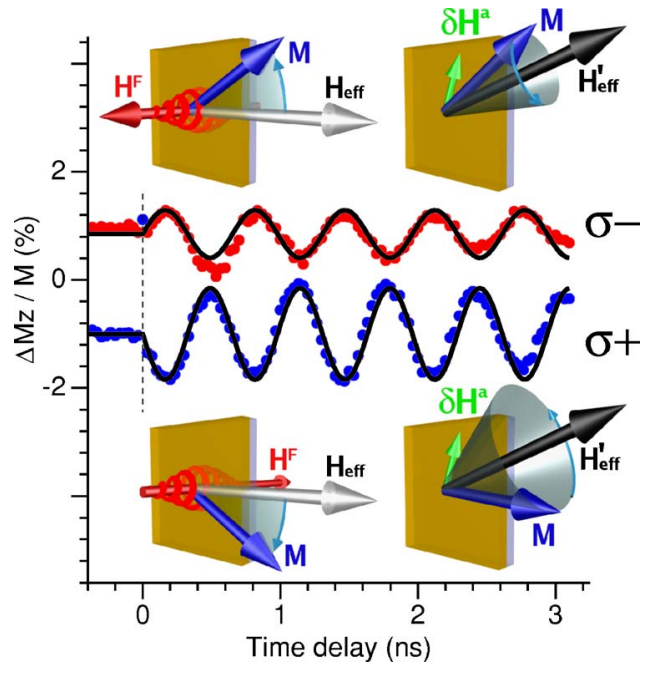

FIG. 8. (Color online) Precession following excitation with circularly polarized light. The two helicities $\sigma^{+}$and $\sigma^{-}$give rise to precession with an opposite phase and a different amplitude. During the $100 \mathrm{fs}$ presence of the laser pulse the magnetization precesses in the dominating axial magnetic field $\mathbf{H}_{\mathrm{F}}$ created by the circularly polarized pump pulse. Subsequent precession takes place in the effective magnetic field $\mathbf{H}_{\text {eff }}^{\prime}=\mathbf{H}_{\text {eff }}+\delta \mathbf{H}^{\mathrm{a}}$.

helicities. Hence, a different effect is needed in order to explain the experimental observations, and the effect that we are seeking should be odd with respect to the helicity of light.

Our experimental observations can be understood if during the presence of the laser pulse a strong magnetic field along the $\mathbf{k}$ vector of light is created. Such an axial magnetic field $\mathbf{H}^{\mathrm{F}}$ can be generated by intense circularly polarized light through what is known as the inverse Faraday effect ${ }^{9,67-69}$ (see below). In our experiment these optically generated field pulses are much stronger than both anisotropy $\mathbf{H}_{\mathrm{a}}$ and the applied field $\mathbf{H}_{\mathrm{ext}}$ and therefore completely dominate during the $\Delta t=100 \mathrm{fs}$ presence of the laser pulse. The magnetization responds by precessing in the plane of the film (normal to $\mathbf{H}^{\mathrm{F}}$ ) to a different in-plane orientation. After the pulse is gone, the magnetization will precess in the effective in-plane field $\mathbf{H}_{\mathrm{eff}}^{\prime}=\mathbf{H}_{\mathrm{ext}}+\mathbf{H}_{\mathrm{a}}+\delta \mathbf{H}^{\mathrm{a}}$, as illustrated in Fig. 8.

The strength of the photoinduced field $\mathbf{H}^{F}$ can be estimated from the precession amplitude $\Lambda$,

$$
H^{F} \approx \frac{\omega}{\gamma} \approx \frac{\Lambda}{\gamma \Delta t_{\text {pulse }}},
$$

where $\omega$ is the precession frequency, $\gamma$ is the gyromagnetic ratio and $\Delta t_{\text {pulse }}$ is the duration of the optical pulse. We find that laser pulses of energy $20 \mu \mathrm{J}$ create transient magnetic field pulses of about $0.6 \mathrm{~T}$ in the garnet films.

The asymmetry seen in the signal amplitude between the $\sigma^{+}$and $\sigma^{-}$helicities stems from the simultaneously created photoinduced anisotropy $\delta \mathbf{H}^{\mathrm{a}}$ which is independent of the pump helicity (see Fig. 8). For the $\sigma^{-}$helicity, M precesses in the direction of the optically modified effective field $\mathbf{H}_{\text {eff }}^{\prime}$ during the presence of $\mathbf{H}^{\mathrm{F}}$. This gives rise to a precession

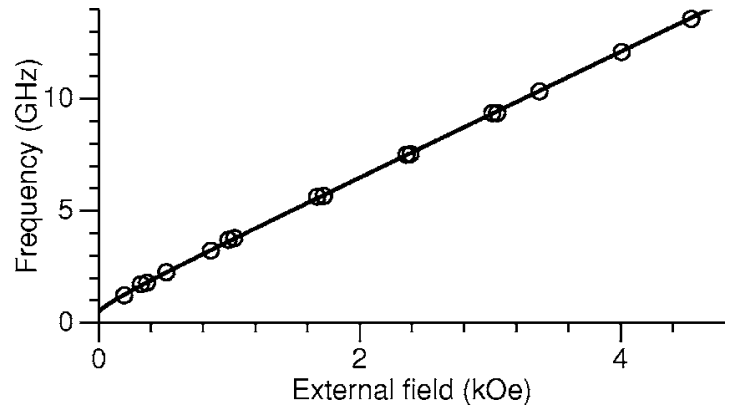

FIG. 9. Precession frequency as function of the externally applied magnetic field. Circles represent measurements and the solid line is a best fit using $4 \pi M_{s}=550 \mathrm{G}$ and $H_{a}=50$ Oe.

with a small amplitude around $\mathbf{H}_{\text {eff }}^{\prime}$ after the pulse is gone. For the $\sigma^{+}$helicity, $\mathbf{M}$ precesses in the opposite direction during the presence of $\mathbf{H}^{\mathrm{F}}$, moving further away from $\mathbf{H}_{\text {eff }}^{\prime}$. After the pulse is gone a large amplitude precession takes place.

The consistently large amplitude precession triggered by $\sigma^{+}$polarized pump pulses, irrespective of the applied field strength $\mathbf{H}_{\text {ext }}$, allows the external field dependence of the precession frequency $\omega\left(H_{\text {ext }}\right)$ to be accurately determined from the experimental data. As will be discussed in Sec. III C, this is not the case for $\sigma^{-}$polarized excitation, which under certain conditions does not trigger any precession (see Fig. 13). The precession frequency is given by the Kittel formula ${ }^{70}$ and can, for our geometry, be expressed as

$$
\omega=\gamma \sqrt{B H}=\gamma \sqrt{\left(4 \pi M_{s}+H_{\mathrm{a}}^{\prime}+H_{\mathrm{ext}}\right)\left(H_{\mathrm{a}}^{\prime}+H_{\mathrm{ext}}\right)},
$$

where the small photoinduced modification $\delta \mathbf{H}^{\mathrm{a}}$ of the anisotropy field has been included in $H_{\mathrm{a}}^{\prime}=H_{\mathrm{a}}+\delta H^{\mathrm{a}}$. Figure 9 shows the measured $\omega$ as a function of the applied magnetic field for the $\sigma^{+}$polarized pump excitation. The solid line represents a best fit using Eq. (18) and gives an $\mathbf{H}_{\mathrm{a}}$ of about $50 \mathrm{Oe}$, in accordance with the results of Fig. 2.

\section{Double-pump coherent magnetization control}

Ultrafast coherent control of the magnetization can be achieved by using multiple laser pulses in rapid succession. In a double pump experiment employing two circularly polarized pump pulses with opposite helicity and almost equal power, we achieved stopping of the precessional dynamics as well as doubling of the amplitude. As already described in the case of linearly polarized pulses, we operate at a fixed time-delay between the two pump pulses, and adjust the frequency of precession by an external magnetic field in order to vary the arrival time of the second pump pulse with respect to the phase of the already present precession.

In Fig. 10 it is shown how a pump pulse of helicity $\sigma^{+}$ arriving at $t=0$ triggers precession of the magnetization, as explained in the previous section. A second pump pulse of helicity $\sigma^{-}$arriving after an odd number of half precessional periods rotates the magnetization further away from $\mathbf{H}_{\text {eff }}$ causing the subsequent precession to have almost twice the amplitude. If, however, this second pump pulse arrives after an integer number of full periods, the magnetization is ro- 


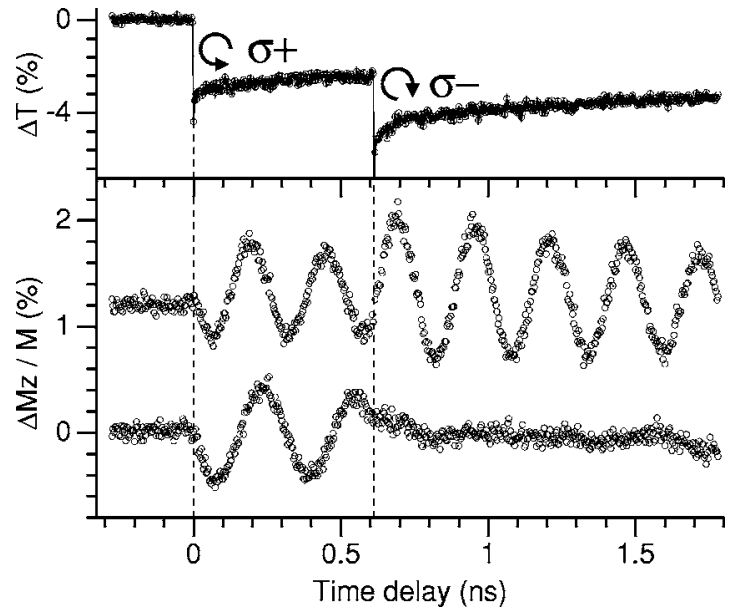

FIG. 10. Double pump experiment with circularly polarized laser pulses of opposite helicity and $15 \mu \mathrm{J}$ pulse power. The upper panel shows the pump-induced change of the sample transmittivity due to the photoexcitation of impurities. The lower panel shows how amplification and complete stopping of the magnetization precession can be achieved depending on the phase of the precession when the second laser pulse arrives. The time delay between the two pump pulses is fixed at approximately $0.6 \mathrm{~ns}$, and the precession frequency is controlled by varying the external field.

tated back into its original equilibrium orientation along $\mathbf{H}_{\text {eff }}$ and no further precession takes place. Figure 11 gives a pictorial illustration of these two situations.

This experiment clearly demonstrates that femtosecond optical pulses can be used to directly and coherently control spin dynamics. Depending on the phase of the precession when the second pulse arrives, energy is either transferred from the laser pulse to the magnetic system (amplification of the precession) or from the magnetic excitation to the optical pulse (stopping of the precession). A stimulated Raman process of scattering on magnons is believed to be responsible for the inverse Faraday effect ${ }^{71}$ (see below), and we expect that further support for this mechanism can be found in the
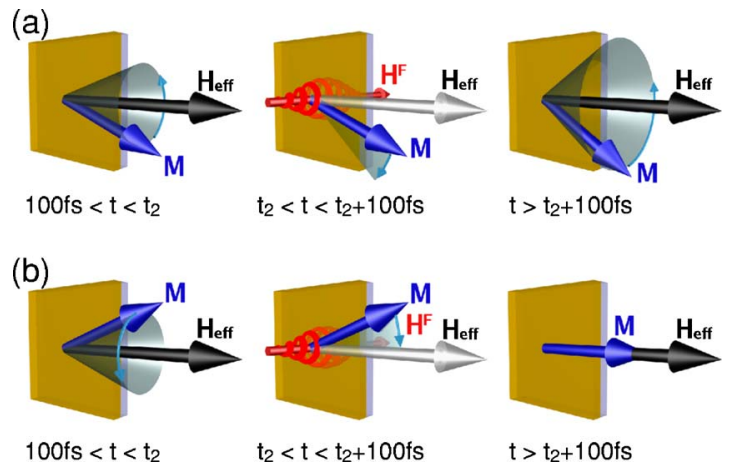

FIG. 11. (Color online) Illustration of the double pump experiment for circularly polarized pump pulses of opposite helicity arriving at an (a) odd number of half precessional periods and (b) an integer number of full precessional periods. The magnetization is either rotated further away from the effective field direction causing subsequent precession to take place with almost twice the original amplitude, or the magnetization is rotated back into the effective field direction and no further precession takes place. frequency spectrum of the second pump pulse. Stokes or anti-Stokes peaks should be observable in the spectrum, depending on whether the precession is amplified or stopped, respectively. In view of the low intrinsic damping in these garnet films, and therefore the long lifetime of magnetic excitations, it is remarkable how ultrashort laser pulses can completely stop the long period coherent precession of spins instantaneously by transfer of the energy into the optical pulse. This process can also be viewed as coherent laser cooling of magnons.

It should be pointed out that the present double pump experiment, which demonstrates control of the magnetization in ferrimagnetic garnets, is considerably different from those previously reported in diamagnetic and paramagnetic materials. During the past two decades a great number of publications have been devoted to the photoexcitation of a nonequilibrium spin polarization in direct band gap semiconductors through the phenomena of optical orientation. ${ }^{72-74}$ In these materials, absorption of circularly polarized photons may lead to a nonequilibrium population of spin polarized electrons and holes in the conduction band and valence band, respectively. In paramagnetic semiconductors these spin polarized carriers can cause partial alignment of the moments of magnetic ions due to a $s p-d$ exchange interaction, and thereby also affect their precession in a magnetic field. ${ }^{75}$ Using this phenomena of optical orientation Akimoto et $a l .{ }^{76}$ have demonstrated control of the precession of $\mathrm{Mn}^{2+}$ moments in $\mathrm{CdTe} / \mathrm{Cd}_{1-x} \mathrm{Mn}_{x} \mathrm{Te}$ quantum wells. Note that this approach, in contrast to our experiment, is based on the absorption of photons. A nonabsorptive mechanism for manipulation of spins in $\mathrm{Zn}_{1-x} \mathrm{Cd}_{x} \mathrm{Se}$ quantum well structures was reported by Gupta et al. ${ }^{77}$ who used below band gap optical pulses to control the spin precession of photoexcited electrons in the conduction band via the optical Stark effect. However, these experiments were performed on paramagnetic materials, while in the present case we have succeeded to control the collective motion of the strongly coupled spins in a magnetically ordered compound. Additionally, the experimental conditions differ strongly in the two cases: control of the spin precession in paramagnetic semiconductors requires very low temperatures, typically below $10 \mathrm{~K}$, and strong magnetic fields of several teslas. In strong contrast, the optical control of magnetization that we report here was done at room temperature and in magnetic fields well below $1 \mathrm{kOe}$.

\section{Phenomenological model of the inverse Faraday effect}

The normal magneto-optical Faraday effect can be viewed as due to a difference in the refractive indices for the two circularly polarized eigenmodes of light propagating in a magnetized medium. The inverse process, where circularly polarized light creates a magnetization or an effective magnetic field is also possible $9,68,69$ and known as the inverse Faraday effect. Strictly speaking this effect is classified as a optomagnetic effect as it does not rely on absorption. ${ }^{47}$ Phenomenologically the creation of an axial magnetic field by circularly polarized light can be described as

$$
\delta H_{i}^{\mathrm{F}}(0)=\chi_{i j k}\left[E_{j}(\omega) E_{k}^{*}(\omega)-E_{k}(\omega) E_{j}^{*}(\omega)\right],
$$

where $\chi_{i j k}$ is a third rank axial tensor with nonzero components for crystals of any symmetry. ${ }^{57}$ The magnetic field is 
created by elliptically or circularly polarized light along its $\mathbf{k}$-vector. The field changes sign when the circular polarization is changed from left-handed to right-handed. The effect does not rely on absorption but becomes possible due to strong spin-orbit coupling in a material. The optically induced magnetic field pulse appears to act only during the presence of the laser pulse in the material. ${ }^{9}$ Its strength depends on the value of the relevant $\chi_{i j k}$ components and is directly related to the Verdet constant. For our garnet films we can estimate the optically induced effective field strength from the resulting precession dynamics. At the wavelength of $805 \mathrm{~nm}$ used in our experiments we find $\mathrm{H}^{\mathrm{F}} \approx 0.6 \mathrm{~T}$ for a pump irradiance of about $10^{11} \mathrm{~W} / \mathrm{cm}^{2}$.

\section{Microscopic model of the inverse Faraday effect}

In this section we discuss a possible microscopic mechanism for the creation of an axial magnetic field by light and argue that it can be both efficient and ultrafast.

In the electric dipole approximation an optical transition cannot change the spin state of an electron. After electric dipole transitions the next most likely type of transition is a magnetic dipole transition, which is due to the interaction between the electron spin and the oscillating magnetic field of the incident electromagnetic radiation. Magnetic dipole transitions allow spin flip but typically are about $10^{5}$ times less probable than similar electric dipole transitions. The strong effect that we see indicates a mechanism that allows change of the electron spin with higher efficiency than expected from a magnetic dipole transition. Moreover, the mechanism should not rely on material properties specific to garnets, as the reported effect has also been shown to exist in other magnetic materials such as rare earth orthoferrites ${ }^{9}$ and metallic alloys. ${ }^{36}$

A stimulated Raman-like coherent optical scattering process has been suggested to account for both the speed and the efficiency of the excitation., ${ }^{9,68,71,78}$ Two frequency components of electromagnetic radiation, both present in the 100-fs-wide laser pulse take part in the process (see Fig. 12). The frequency $\omega_{1}$ stimulates an optical transition from the ground state $|1\rangle$ to a virtual state with a strong spin-orbit interaction. Due to this strong spin-orbit coupling there is a large probability of flipping the electron spin. Radiation at the frequency $\omega_{2}$ also present in the optical pulse, stimulates the relaxation back into the spin split ground state with the electron spin reversed. The relaxation is accompanied by the coherent emission of a photon of energy $\hbar\left(\omega_{1}-\Omega_{m}\right)$ and the creation of a magnon of energy $\Omega_{\mathrm{m}}$. This process can be much more efficient than a simple magnetic dipole transition as it is coherently stimulated by radiation at a frequency of $\omega_{2}$ present in the laser pulse. Moreover, as the energy of the virtual state is of the order of the photon energy $E=\hbar \omega=1.54 \mathrm{eV}$ the transition can be fast, of the order of $\tau \sim h / E \sim 3$ fs.

\section{Single-pump ultrafast photomagnetic switching}

A proper combination of the inverse Faraday effect and the photoinduced anisotropy allows for an interesting demonstration of photomagnetic switching on the femtosecond

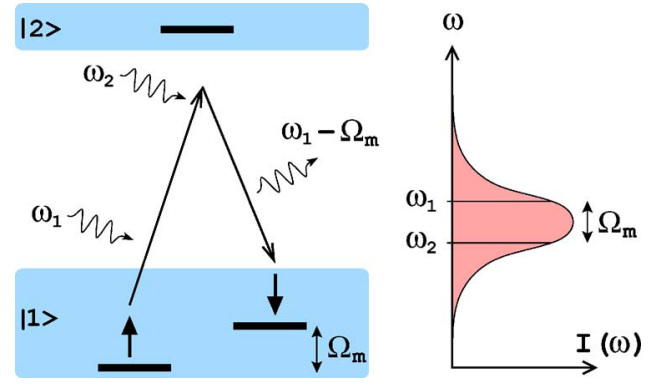

FIG. 12. (Color online) Illustration of the stimulated Raman-like coherent scattering mechanism believed to be responsible for the ultrafast optically generated magnetic field. Two frequency components of electromagnetic radiation from the spectrally broad laser pulse take part in the process. The frequency $\omega_{1}$ causes a transition into a virtual state with strong spin-orbit coupling. Radiation at the frequency $\omega_{2}$ stimulates the relaxation back to the ground state with the creation of a magnon.

time scale. ${ }^{48}$ Because the direction of $\delta \mathbf{H}^{\mathrm{a}}$ depends only on the initial angle $\phi$ of the magnetization with respect to the crystal axes, it can be tuned by rotating the sample with respect to the applied field. We have verified experimentally that this is the case. Alternatively, since the initial equilibrium of $\mathbf{M}$ is along $\mathbf{H}_{\mathrm{eff}}$, which is determined by the balance between the magnetocrystalline anisotropy field $\mathbf{H}_{\mathrm{a}}$ and the externally applied field $\mathbf{H}_{\text {ext }}$, it can also be tuned simply by varying the strength of the applied field.

In Fig. 13 the coherent precession of the magnetization following excitation with pulses of helicity $\sigma^{-}$and $\sigma^{+}$is shown for different values of $\mathbf{H}_{\mathrm{ext}}$. The amplitude of precession is consistently larger in the case of $\sigma^{+}$, as during $0<t<100 \mathrm{fs}, \mathbf{M}$ precesses away from the new equilibrium created by $\delta \mathbf{H}^{\mathrm{a}}$, as explained above in Sec. III B 1 . For pulses of helicity $\sigma^{-}$, this precession is towards the new equilibrium, leading to smaller precessional amplitude in the time after the pulse. With an applied field of $\left|\mathbf{H}_{\text {ext }}\right| \approx 150 \mathrm{Oe}$, no precession is triggered due to a perfect balance of two effects. The in-plane precession of the magnetization during the 100 fs magnetic field pulse $\delta \mathbf{H}^{\mathrm{F}}$ brings the magnetization exactly to the new equilibrium orientation created by the optically modified anisotropy field. It remains stable in this orientation until the anisotropy field relaxes back to its original state, i.e., for several nanoseconds. An illustration of this switching process is shown in Fig. 14.

Note also that for the $\sigma^{-}$helicity at weak applied fields the precession has an opposite phase compared to the precession in stronger applied fields, and that this phase is the same as for the precession triggered by the $\sigma^{+}$pulses. At weak fields the direction of the photoinduced $\delta \mathbf{H}^{\mathrm{a}}$ is such that the precession of $\mathbf{M}$ in $\mathbf{H}^{\mathrm{F}}$ during the optical pulse is not sufficient to bring it into the direction of $\mathbf{H}_{\text {eff }}^{\prime}$. At stronger fields, however, $\delta \mathbf{H}^{\mathrm{a}}$ is in a different direction producing a $\mathbf{H}_{\mathrm{eff}}^{\prime}$ that is less inclined with respect to the original effective field. During the presence of $\mathbf{H}^{\mathrm{F}}$ the magnetization now precesses past the direction of $\mathbf{H}_{\mathrm{eff}}^{\prime}$, and therefore with the opposite phase in the time after the laser pulse.

\section{Temperature dependence}

The experiments described so far were all done at room temperature and mostly in the in-plane applied field geom- 


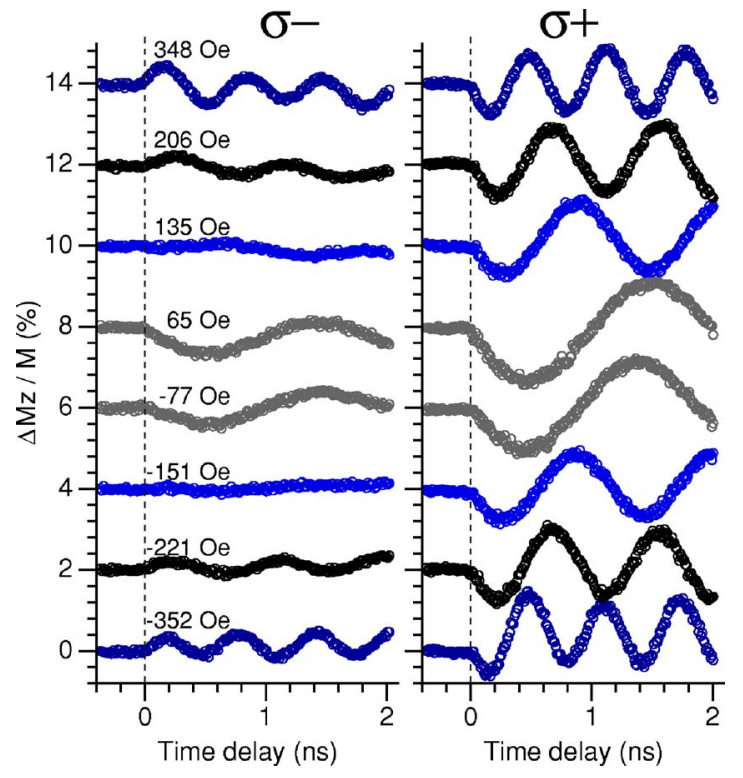

FIG. 13. (Color online) Precession of the magnetization triggered by left- and right-handed circularly polarized laser pulses at different values of the in-plane applied magnetic field. For the $\sigma^{-}$ helicity, at an applied field of $\sim \pm 150$ Oe, no precession is observed due to a perfect balance of the two photomagnetic effects $\delta \mathbf{H}^{\mathrm{a}}$ and $\mathbf{H}^{\mathrm{F}}$.

etry, where thermal effects of the laser excitation would not be clearly visible even if it were present. In a tilted field geometry $\left(\zeta<90^{\circ}\right)$ the measured signal is more sensitive to thermal effects of the laser pumping, as the equilibrium orientation of $\mathbf{M}$ is determined by both the anisotropy field and the magnitude of M. A thermal influence on any of these would alter the equilibrium orientation for $\mathbf{M}$ and thus trigger precessional dynamics or spin reorientation.

The phonon-magnon interaction time in dielectric materials is typically of the order of a nanosecond. ${ }^{44}$ Heating can therefore not account for the ultrafast $(<1 \mathrm{ps})$ excitation of coherent spin waves that we have shown. However, thermal effects induced by the laser pulses may still be present but on a longer time scale.

In our experiment, the Faraday rotation of the probe pulses is proportional to $M_{z}$. For magnetization in the film

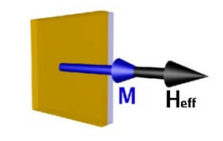

Initial state $\mathrm{t}<0$

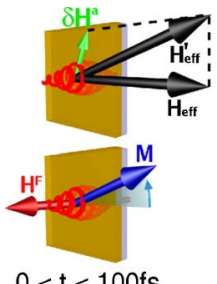

$0<t<100$ fs

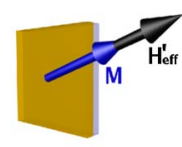

Final state $t>100$ fs
FIG. 14. (Color online) Illustration of the switching process. Initially at $t<0$ the magnetization is along $\mathbf{H}_{\text {eff }}$. During the presence of the laser pulse $0<t<100$ fs photo induced modification of the anisotropy fields leads to a new long-lived equilibrium along $\mathbf{H}_{\text {eff }}^{\prime}$. Simultaneously, the strong optically generated field $\mathbf{H}^{\mathrm{F}}$ causes the magnetization to precess into this new state. After $t>100 \mathrm{fs}$, the optical pulse is gone and the approximately $0.6^{\circ}$ switching of $\mathbf{M}$ is complete.

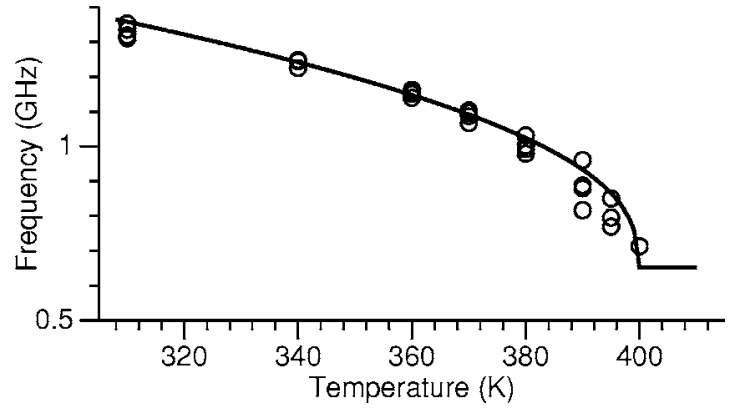

FIG. 15. Precession frequency as a function of the temperature for an in-plane applied magnetic field of about $250 \mathrm{Oe}$ and $\sigma^{-}$polarized excitation. The symbols represent measurements and the solid line the theoretical prediction with anisotropy $H_{a}=50 \mathrm{Oe}$ and an assumed temperature dependence for $H_{a}$ like $M(T)^{2}$.

plane $\left(\zeta=90^{\circ}\right)$ this means that the measured signal is proportional to the magnetization $M(T)$ at any given temperature. In a tilted field geometry $\left(\zeta<90^{\circ}\right)$ the signal is not simply proportional to $M(T)$ but to $M_{z}(T)$ which is given by the balance $\mathbf{M} \times \mathbf{H}_{\text {eff }}=0$ in the effective magnetic field $\mathbf{H}_{\mathrm{eff}}=-4 \pi M_{z} \hat{\mathbf{z}}+\mathbf{H}_{\mathrm{a}}+\mathbf{H}_{\mathrm{ext}}$.

In order to investigate the thermal effects the sample was mounted on a temperature controlled sample holder and laser-induced magnetization dynamics was studied at temperatures from $T=300 \mathrm{~K}$ to $T=410 \mathrm{~K}$. In Fig. 15 it is shown how the precession frequency depends on the sample temperature with an applied field $\mathbf{H}_{\text {ext }}$ of about $250 \mathrm{Oe}$ in the sample plane. The solid line represents a fit using Eq. (18) with $\left|\mathbf{H}_{\mathrm{a}}\right| \propto M^{2}(T)$ assumed to be the temperature dependence of the magnetocrystalline anisotropy. This is in good agreement with the measured precession frequencies.

The precession amplitude was measured as a function of temperature for both the linear and circular polarization of the pump laser. The results are shown in Fig. 16. When excited with linearly polarized light, the precession amplitude drops quickly with temperature and is no longer visible at $380 \mathrm{~K}$, implying that the efficiency of the photoinduced anisotropy $\delta \mathbf{H}^{\mathrm{a}}$ is strongly temperature dependent. This observation is in qualitative agreement with the temperature dependence of the density of photoproduced charges in garnets, ${ }^{79}$ a process necessary for the light-induced change of magnetic anisotropy.

In contrast, the amplitude of the precession excited with circularly polarized light has a very different dependence on $T$. It also appears to behave differently for the two helicities $\sigma^{+}$and $\sigma^{-}$. As described in the previous section, the difference in amplitude between $\sigma^{+}$and $\sigma^{-}$stems from the contribution by $\delta \mathbf{H}^{\mathrm{a}}$, whose temperature dependence is shown in panel (a). The effect of photoinduced anisotropy $\delta \mathbf{H}^{\mathrm{a}}$ can be eliminated by averaging the curves for $\sigma^{+}$and $\sigma^{-}$. The resulting average values fit well to a scaled $M(T)$ curve [see panel (c)]. Note that the measured signal amplitude in this geometry is expected to be proportional to $M(T)$ and that this result therefore implies that the inverse Faraday effect $\mathbf{H}^{\mathrm{F}}$ is independent of $\mathbf{M}$, in accordance with the phenomenological description given in Eq. (19).

In order to determine the amount of heating caused by a single pump pulse and to estimate the phonon-magnon inter- 


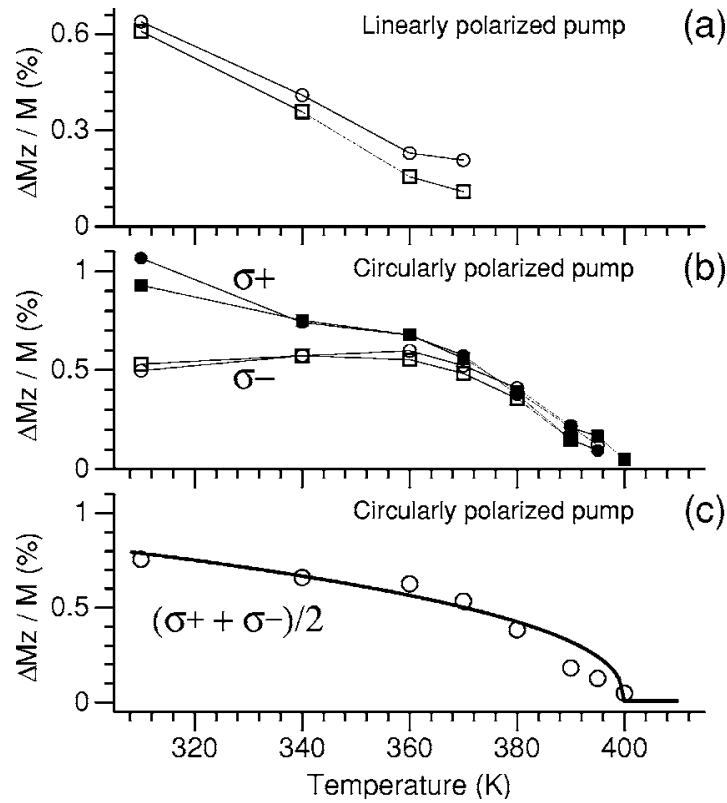

FIG. 16. Amplitude of the precession triggered by (a) linearly polarized pump pulses and (b) circularly polarized pulses of helicity $\sigma^{+}$and $\sigma^{-}$. (c) The average of the $\sigma^{+}$and $\sigma^{-}$amplitudes from (b) which eliminates the contribution from $\delta \mathbf{H}^{\mathrm{a}}$. This averaged amplitude follows an $M(T)$ temperature dependence, implying that $\mathbf{H}^{\mathrm{F}}$ is independent of $\mathbf{M}$.

action time, the laser-induced magnetization dynamics was studied in a tilted field geometry $\left(\zeta<90^{\circ}\right)$ at different temperatures. The results are shown in Fig. 17. In panel (a), the time traces of the precession are shown for sample temperatures from 310 up to $410 \mathrm{~K}$. As the temperature approaches the Curie point $T_{\mathrm{C}}$, the amplitude of precession decreases and an exponential background appears. This is the thermal destruction of the magnetic order induced by the laser heating. The time constant is of the order of a nanosecond and determined by the phonon-magnon interaction time. ${ }^{44}$

From the time traces in Fig. 17(a) the slope of the exponential background was extracted. This is shown in panel 17 (c) as a function of the temperature. The solid line is the function $M_{z}(T+\Delta T)-M_{z}(T)$ with $M_{z}(T)$ taken from the measurements shown in panel 17(b). A best fit is obtained for a pump-induced temperature increase $\Delta T=18 \mathrm{~K}$ caused by a laser irradiance of about $10^{11} \mathrm{~W} / \mathrm{cm}^{2}$. When measuring at a repetition rate of $500 \mathrm{~Hz}$ there is no static overheating of the sample. This has been verified by measuring magnetization curves $M(T)$ with and without the presence of pump pulses.

Another point that deserves to be addressed is the significant increase of signal noise seen in the time traces at temperatures from $370 \mathrm{~K}$ to $T_{\mathrm{C}}=400 \mathrm{~K}$. Already at $370 \mathrm{~K}$ and $380 \mathrm{~K}$ near the end of the time traces the noise level starts to increase as heating induced by the laser pulse reaches the magnetic system. Even more pronounced levels of noise can be seen at temperatures closer to the Curie point. This noise is due to the thermal fluctuations in the magnetic system. When studied with our stroboscopic measurement technique that averages every data point over several excitation events,
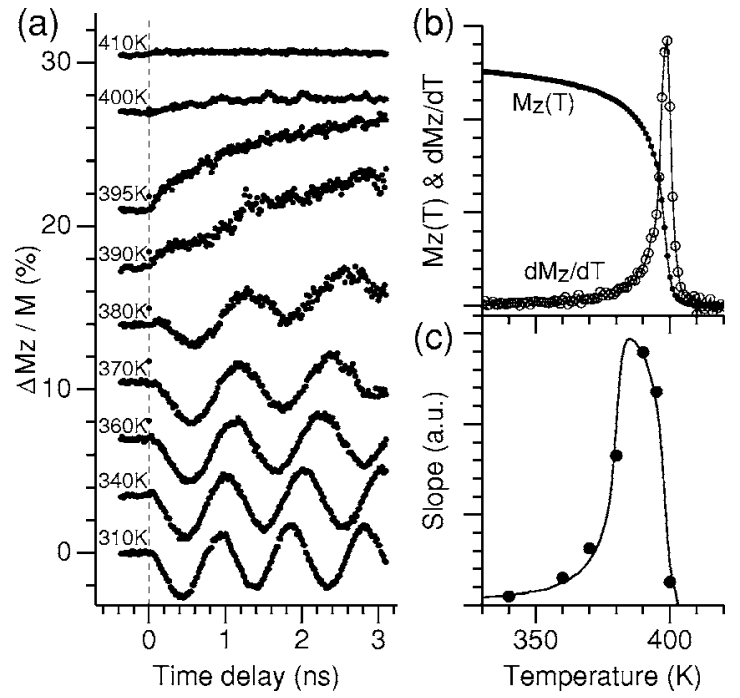

FIG. 17. (a) Precessional dynamics at different temperatures for an applied field $\mathrm{H}_{\mathrm{ext}}=300 \mathrm{Oe}$ at an angle of about $45^{\circ}$. (b) The $z$ component of the magnetization (proportional to the Faraday rotation) and its temperature derivative as function of the sample temperature. (c) The measured slope of the exponential relaxation of $\mathbf{M}$ as a function of temperature. The solid line represents a best fit.

the fluctuations show up as random noise in the signal. ${ }^{80}$ As we approach the critical temperature the fluctuations strongly increase and then drop markedly above $T_{\mathrm{C}}$, where the magnetic order is completely destroyed. The observed behavior of the noise confirms that we are indeed probing the magnetic system.

\section{CONCLUSION}

In conclusion, we have shown that the magnetization in garnet films can be directly and coherently controlled on the femtosecond time scale using ultrashort laser pulses. Two distinct nonthermal photomagnetic effects that facilitate such control have been identified. A long-lived photoinduced magnetic anisotropy field can be created by both linearly and circularly polarized laser pulses, and strong transient magnetic field pulses can be generated by circularly polarized light. Applying a small external field allows for the careful timing and balancing of these two effects, thus making complete nonthermal and coherent control of the magnetization possible. Thermal effects were only seen near $T_{\mathrm{C}}$. They are slow due to a phonon-magnon interaction time of the order of a nanosecond, and can easily be distinguished from the ultrafast nonthermal processes. The reported effects open exciting possibilities for ultrafast manipulation of magnetization by light.

\section{ACKNOWLEDGMENTS}

The authors thank L.E. Helseth and T.H. Johansen for providing the samples. This work was supported by The Norwegian Research Council, The European network DYNAMICS, FOM, and NWO. 
*Electronic address: f.hansteen@science.ru.nl

${ }^{1}$ C. H. Back, D. Weller, J. Heidmann, D. Mauri, D. Guarisco, E. L. Garwin, and H. C. Siegmann, Phys. Rev. Lett. 81, 3251 (1998).

${ }^{2}$ C. H. Back, R. Allenspach, W. Weber, S. S. P. Parkin, D. Weller, E. L. Garwin, and H. C. Siegmann, Science 285, 864 (1999).

${ }^{3}$ Y. Acremann, C. H. Back, M. Buess, O. Portmann, A. Vaterlaus, D. Pescia, and H. Melchior, Science 290, 492 (2000).

${ }^{4}$ Y. Acremann, M. Buess, C. H. Back, M. Dumm, G. Bayreuther, and D. Pescia, Nature (London) 414, 51 (2001).

${ }^{5}$ T. Gerrits, H. A. M. van den Berg, J. Hohlfeld, L. Bär, and Th. Rasing, Nature (London) 418, 509 (2002).

${ }^{6}$ I. Tudosa, C. Stamm, A. B. Kashuba, F. King, H. C. Siegmann, J. Stöhr, G. Ju, B. Lu, and D. Weller, Nature (London) 428, 831 (2004).

${ }^{7}$ C. Stamm, I. Tudosa, H. C. Siegmann, J. Stöhr, A. Yu. Dobin, G. Woltersdorf, B. Heinrich, and A. Vaterlaus, Phys. Rev. Lett. 94, 197603 (2005).

${ }^{8}$ A. V. Kimel, A. Kirilyuk, A. Tsvetkov, R. V. Pisarev, and Th. Rasing, Nature (London) 429, 850 (2004).

${ }^{9}$ A. V. Kimel, A. Kirilyuk, P. A. Usachev, R. V. Pisarev, A. M. Balbashov, and Th. Rasing, Nature (London) 435, 655 (2005).

${ }^{10}$ U. Keller, Nature (London) 424, 831 (2003).

${ }^{11}$ M. B. Agranat, S. I. Ashitkov, A. B. Granovskii, and G. I. Rukman, Zh. Eksp. Teor. Fiz. 86, 1376 (1984), [Sov. Phys. JETP 59, 804 (1984)].

${ }^{12}$ A. Vaterlaus, T. Beutler, and F. Meier, J. Appl. Phys. 67, 5661 (1990)

${ }^{13}$ A. Vaterlaus, T. Beutler, and F. Meier, Phys. Rev. Lett. 67, 3314 (1991).

${ }^{14}$ E. Beaurepaire, J.-C. Merle, A. Daunois, and J.-Y. Bigot, Phys. Rev. Lett. 76, 4250 (1996).

${ }^{15}$ A. Scholl, L. Baumgarten, R. Jacquemin, and W. Eberhardt, Phys. Rev. Lett. 79, 5146 (1997).

${ }^{16}$ J. Hohlfeld, E. Matthias, R. Knorren, and K. H. Bennemann, Phys. Rev. Lett. 78, 4861 (1997).

${ }^{17}$ J. Güdde, U. Conrad, V. Jähnke, J. Hohlfeld, and E. Matthias, Phys. Rev. B 59, R6608 (1999).

${ }^{18}$ E. Beaurepaire, M. Maret, V. Halté, J.-C. Merle, A. Daunois, and J.-Y. Bigot, Phys. Rev. B 58, 12134 (1998).

${ }^{19}$ H. Regensburger, R. Vollmer, and J. Kirschner, Phys. Rev. B 61, 14716 (2000).

${ }^{20}$ B. Koopmans, M. van Kampen, J. T. Kohlhepp, and W. J. M. de Jonge, Phys. Rev. Lett. 85, 844 (2000).

${ }^{21}$ P. M. Oppeneer and A. Liebsch, J. Phys.: Condens. Matter 16, 5519 (2004).

${ }^{22}$ L. Guidoni, E. Beaurepaire, and J.-Y. Bigot, Phys. Rev. Lett. 89, 017401 (2002).

${ }^{23}$ E. Beaurepaire, G. M. Turner, S. M. Harrel, M. C. Beard, J.-Y. Bigot, and C. A. Schmuttenmaer, Appl. Phys. Lett. 84, 3465 (2004).

${ }^{24}$ J.-Y. Bigot, L. Guidoni, E. Beaurepaire, and P. N. Saeta, Phys. Rev. Lett. 93, 077401 (2004).

${ }^{25}$ W. Hübner and G. P. Zhang, Phys. Rev. B 58, R5920 (1998).

${ }^{26}$ A. Vernes and P. Weinberger, Phys. Rev. B 71, 165108 (2005).

${ }^{27}$ G. Ju, A. V. Nurmikko, R. F. C. Farrow, R. F. Marks, M. J. Carey, and B. A. Gurney, Phys. Rev. Lett. 82, 3705 (1999).

${ }^{28}$ G. Ju, L. Chen, A. V. Nurmikko, R. F. C. Farrow, R. F. Marks, M. J. Carey, and B. A. Gurney, Phys. Rev. B 62, 1171 (2000).

${ }^{29}$ M. van Kampen, C. Jozsa, J. T. Kohlhepp, P. LeClair, L. Lagae, W. J. M. de Jonge, and B. Koopmans, Phys. Rev. Lett. 88,
227201 (2002).

${ }^{30}$ Q. Zhang, A. V. Nurmikko, A. Anguelouch, G. Xiao, and A. Gupta, Phys. Rev. Lett. 89, 177402 (2002).

${ }^{31}$ R. Wilks, R. J. Kicken, M. Ali, B. J. Hickey, J. D. R. Buchanan, A. T. G. Pym, and B. K. Tanner, J. Appl. Phys. 95, 7441 (2004).

${ }^{32}$ M. Vomir, L. H. F. Andrade, L. Guidoni, E. Beaurepaire, and J.-Y. Bigot, Phys. Rev. Lett. 94, 237601 (2005).

${ }^{33}$ G. P. Zhang and W. Hübner, Phys. Rev. Lett. 85, 3025 (2000).

${ }^{34}$ G. Ju, A. Vertikov, A. V. Nurmikko, C. Canady, G. Xiao, R. F. C. Farrow, and A. Cebollada, Phys. Rev. B 57, R700 (1998).

${ }^{35}$ J.-Y. Bigot, C. R. Acad. Sci., Ser IV: Phys., Astrophys. 2, 1483 (2001).

${ }^{36}$ C. D. Stanciu, F. Hansteen, A. V. Kimel, A. Tsukamoto, A. Itoh, A. Kirilyuk, and Th. Rasing (unpublished). Recently we have managed to suppress this dominating thermal effect in $\mathrm{GdFeCo}$ and to observe true nonthermal effects.

${ }^{37}$ H. Ohno, Science 281, 951 (1998).

${ }^{38}$ H. Ohno, A. Shen, F. Matsukura, A. Oiwa, A. Endo, S. Katsumoto, and Y. Iye, Appl. Phys. Lett. 69, 363 (1996).

${ }^{39}$ A. Oiwa, Y. Mitsumori, R. Moriya, T. Slupinski, and H. Munekata, Phys. Rev. Lett. 88, 137202 (2002).

${ }^{40}$ Y. Mitsumori, A. Oiwa, T. Slupinski, H. Maruki, Y. Kashimura, F. Minami, and H. Munekata, Phys. Rev. B 69, 033203 (2004).

${ }^{41}$ A. V. Kimel, G. V. Astakhov, G. M. Schott, A. Kirilyuk, D. R. Yakovlev, G. Karczewski, W. Ossau, G. Schmidt, L. W. Molenkamp, and Th. Rasing, Phys. Rev. Lett. 92, 237203 (2004).

${ }^{42}$ E. Kojima, R. Shimano, Y. Hashimoto, S. Katsumoto, Y. Iye, and M. Kuwata-Gonokami, Phys. Rev. B 68, 193203 (2003).

${ }^{43}$ J. Wang, C. Sun, J. Kono, A. Oiwa, H. Munekata, Ł. Cywiński, and L. J. Sham, Phys. Rev. Lett. 95, 167401 (2005).

${ }^{44}$ A. V. Kimel, R. V. Pisarev, J. Hohlfeld, and Th. Rasing, Phys. Rev. Lett. 89, 287401 (2002).

${ }^{45}$ G. Winkler, Magnetic Garnets (Friedr. Vieweg \& Sohn, Braunschweig, Germany, 1981).

${ }^{46}$ Physics of Magnetic Garnets, edited by A. Paoletti, Enrico Fermi International School of Physics, Italian Physical Society (NorthHolland Publishing Co., Amsterdam, 1978), ISBN 0-44485200-x.

${ }^{47}$ A. F. Kabychenkov, Zh. Eksp. Teor. Fiz. 100, 1219 (1991) [Sov. Phys. JETP 73, 672 (1991)].

${ }^{48}$ F. Hansteen, A. V. Kimel, A. Kirilyuk, and Th. Rasing, Phys. Rev. Lett. 95, 047402 (2005).

${ }^{49}$ B. B. Krichevtsov, V. V. Pavlov, and R. V. Pisarev, Zh. Eksp. Teor. Fiz. 49, 466 (1989) [Sov. Phys. JETP 49, 535 (1989)].

${ }^{50}$ R. V. Pisarev, B. B. Krichevtsov, V. N. Gridnev, V. P. Klin, D. Fröhlich, and C. Pahlke-Lerch, J. Phys.: Condens. Matter 5, 8621 (1993).

${ }^{51}$ F. Hansteen, O. Hunderi, T. H. Johansen, A. Kirilyuk, and Th. Rasing, Phys. Rev. B 70, 094408 (2004).

${ }^{52}$ A. Tsukamoto, K. Nakagawa, A. Itoh, A. Kimel, A. Tsvetkov, H. A. N. Ohta, A. Kirilyuk, and Th. Rasing, IEEE Trans. Magn. 40, 2543 (2004).

${ }^{53}$ L. Landau and E. Lifshitz, Phys. Z. Sowjetunion 8, 153 (1935).

${ }^{54}$ Spin Dynamics in Confined Magnetic Structures I, edited by B. Hillebrands and K. Ounadjela, Topics in Applied Physics (Springer, Berlin, 2002).

${ }^{55}$ Y. R. Shen, The Principles of Nonlinear Optics (Wiley, New York, 1984).

${ }^{56}$ T. H. O'Dell, The Electrodynamics of Magneto-electric Media (North-Holland Publishing Company, Amsterdam, 1970). 
${ }^{57}$ R. R. Birss, Symmetry and Magnetism, 2nd ed., Series of monographs on selected topics in solid state physics (North-Holland Publishing Company, Amsterdam, 1966).

${ }^{58}$ V. V. Pavlov, R. V. Pisarev, A. Kirilyuk, and Th. Rasing, Phys. Rev. Lett. 78, 2004 (1997)

${ }^{59}$ R. W. Teale and D. W. Temple, Phys. Rev. Lett. 19, 904 (1967).

${ }^{60}$ J. F. Dillon, E. M. Gyorgy, and J. P. Remeika, Phys. Rev. Lett. 22, 643 (1969).

${ }^{61}$ A. B. Chizhik, I. I. Davidenko, A. Maziewski, and A. Stupakiewicz, Phys. Rev. B 57, 14366 (1998).

${ }^{62}$ A. Stupakiewicz, A. Maziewski, I. Davidenko, and V. Zablotskii, Phys. Rev. B 64, 064405 (2001).

${ }^{63}$ R. Alben, E. M. Gyorgy, J. F. Dillon, and J. P. Remeika, Phys. Rev. B 5, 2560 (1972).

${ }^{64}$ V. G. Veselago, R. A. Doroshenko, and S. G. Rudov, Zh. Eksp. Teor. Fiz. 105, 638 (1994), [JETP 78, 341 (1994)].

${ }^{65}$ R. Metselaar, M. A. H. Huyberts, and H. Logmans, J. Appl. Phys. 46, 7326 (1975).

${ }^{66}$ V. V. Eremenko, S. L. Gnatchenko, I. S. Kachur, V. G. Piryatinskaya, A. M. Ratner, and V. V. Shapiro, Phys. Rev. B 61, 10670 (2000).

${ }^{67}$ L. P. Pitaevskii, Sov. Phys. JETP 12, 1008 (1961).

${ }^{68}$ P. S. Pershan, J. P. van der Ziel, and L. D. Malmstrom, Phys. Rev. 143, 574 (1966).

${ }^{69}$ J. P. van der Ziel, P. S. Pershan, and L. D. Malmstrom, Phys. Rev.
Lett. 15, 190 (1965).

${ }^{70}$ C. Kittel, Phys. Rev. 73, 155 (1948).

${ }^{71}$ Y. R. Shen and N. Bloembergen, Phys. Rev. 143, 372 (1966).

${ }^{72}$ Optical Orientation, edited by F. Meier and B. P. Zakharchenya of Modern Problems in Condensed Matter Sciences Vol. 8 (North-Holland, Amsterdam, 1984), ISBN 0-444-86741-4.

${ }^{73}$ D. D. Awschalom, J. Warnock, and S. von Molnár, Phys. Rev. Lett. 58, 812 (1987).

${ }^{74}$ I. Zutic, J. Fabian, and S. Das Sarma, Rev. Mod. Phys. 76, 323 (2004).

${ }^{75}$ Diluted Magnetic Semiconductors, edited by J. K. Furdyna and J. Kossut, Semiconductors and Semimetals Vol. 25 (Academic Press, New York, 1988), ISBN 0-12-752125-9.

${ }^{76}$ R. Akimoto, K. Ando, F. Sasaki, S. Kobayashi, and T. Tani, J. Appl. Phys. 84, 6318 (1998).

${ }^{77}$ J. A. Gupta, R. Knobel, N. Samarth, and D. D. Awschalom, Science 292, 2458 (2001).

${ }^{78}$ R. Gómez-Abal, O. Ney, K. Satitkovitchai, and W. Hübner, Phys. Rev. Lett. 92, 227402 (2004).

${ }^{79}$ V. V. Eremenko, S. L. Gnatchenko, I. S. Kachur, V. G. Piryatinskaya, A. M. Ratner, M. B. Kosmyna, B. P. Nazarenko, and V. M. Puzikov, J. Phys.: Condens. Matter 15, 4025 (2003).

${ }^{80}$ M. R. Freeman, G. M. Steeves, G. E. Ballentine, and A. Krichevsky, J. Appl. Phys. 91, 7326 (2002). 